\title{
Highly luminescent hetero-ligand MOF nanocrystals with engineered massive Stokes shift for photonic applications.
}

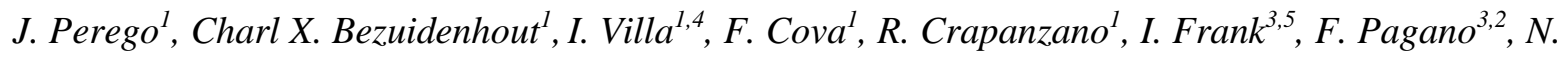

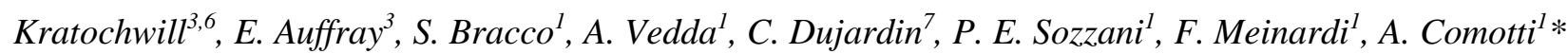 \\ and A. Monguzzi $i^{1 *}$
}

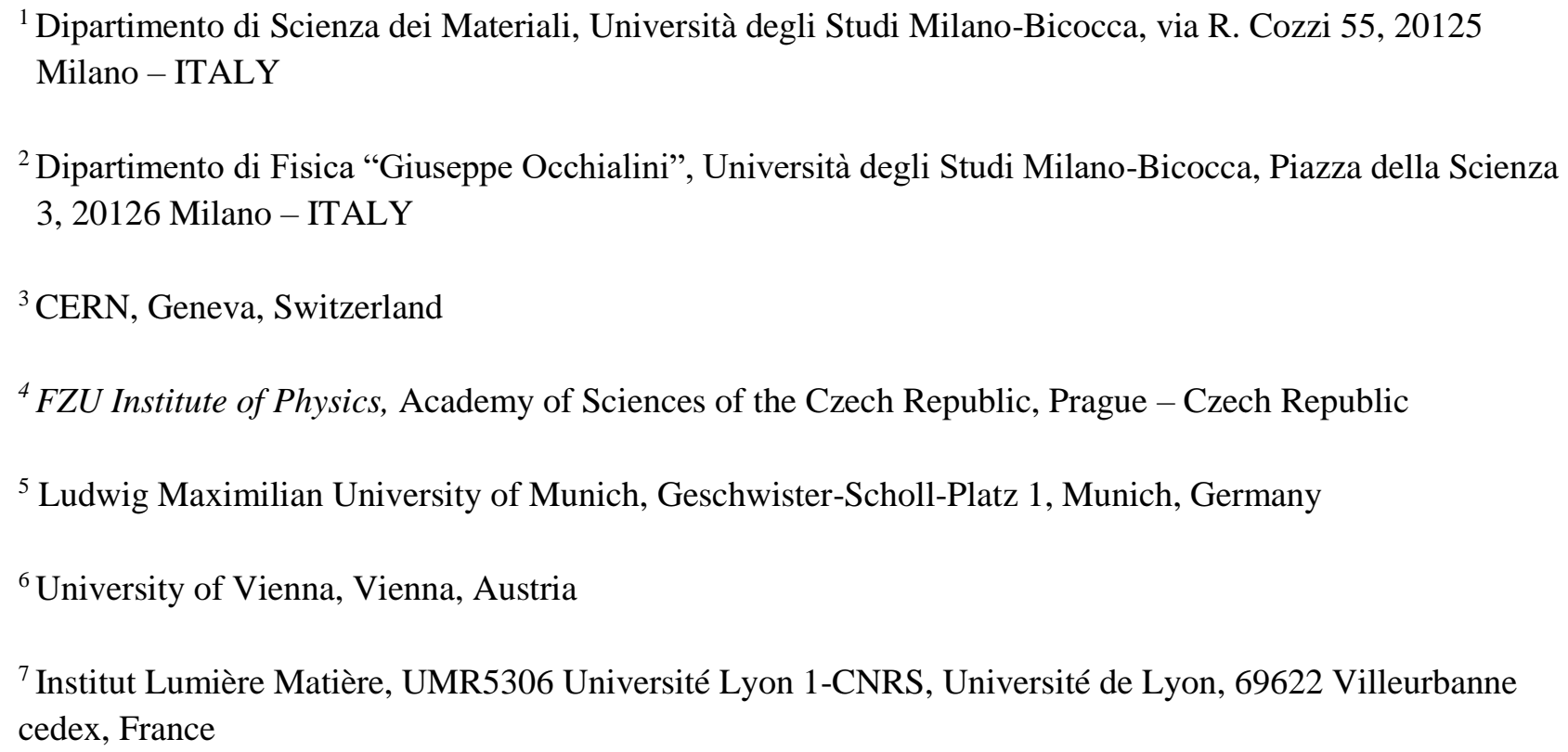

\section{Abstract}

An high efficiency emission with a massive Stokes shift is obtained by fluorescent conjugated acene building blocks arranged in nanocrystals. The two ligands of equal molecular length and connectivity, yet complementary electronic properties, are co-assembled by zirconium oxy-hydroxy clusters, generating highly crystalline hetero-MOF nanoparticles The fast diffusion of singlet molecular excitons in the framework, coupled with the fine matching of ligands absorption and emission properties, enables to achieve an ultrafast activation of the low energy emission by diffusion-mediated non-radiative energy transfer in the 100 ps time scale, by using a low amount of co-ligands. This allow to obtain MOF nanocrystals with a fluorescence quantum efficiency of $\sim 70 \%$ and an actual Stokes shift as large as $750 \mathrm{meV}$. This large Stokes shift suppresses the reabsorption of fast emission issues in bulk devices, pivotal for a plethora of applications in photonics and photon managing spacing from solar technologies, imaging, and detection of high energy radiation. These features allowed to realize a prototypal fast nanocomposite scintillator that shows an enhanced performance with respect to the homo-ligand nanocrystals, achieving benchmark. values which compete with those of some inorganic and organic commercial systems. 
The Stokes shift is an important property of luminescent materials, defined as the energy difference $(\Delta E)$ between the absorption band maximum and the emission spectrum maximum frequencies. ${ }^{1}$ The value of $\Delta E$ is a key parameter in photonic devices and applications because, at a first approximation, it enables to estimate if a specific emitter would be affected by significant reabsorption of the generated light. For example, if the $\Delta E$ value is lower or similar to the bandwidth of the absorption and emission spectra, the consequent intrinsic extensive 'inner-filter' effect can heavily limit the lighting performance of bulk photonic devices, and, in the worst cases, it can also affect the kinetics of the luminescence generation. ${ }^{2-4}$ Conversely, if $\Delta E$ is larger than the spectral bandwidths the system can be considered a large Stokes shift emitter, with no inner filter effects (Fig.1a). These reabsorptionfree materials are highly desirable for several applications. For example, in fluorescence imaging large Stokes shift optical probes allow to obtain high contrast images with limited excitation stray light, avoiding the use of expensive filtering component or time-consuming image post-processing. ${ }^{5,6}$ For solar applications, large Stokes shift emitters are undoubtedly the most promising materials to realize luminescent solar concentrators without reabsorption of the condensed radiation. ${ }^{7}$ Similarly, the sensitivity of scintillating detectors for ionizing radiation would greatly benefit from the use of fast emitters with no reabsorption ${ }^{8}$ showing good light output intensity without effects on the scintillation pulse timing, as required by the most advanced medical imaging techniques such as timeof-flight positron emission tomography (TOF-PET) ${ }^{9}$ and high-rate high-energy physics (HEP) experiments.

a

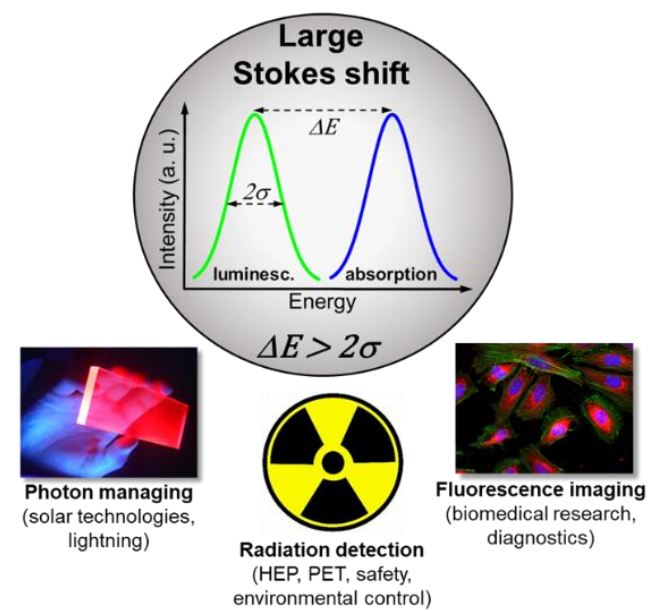

b

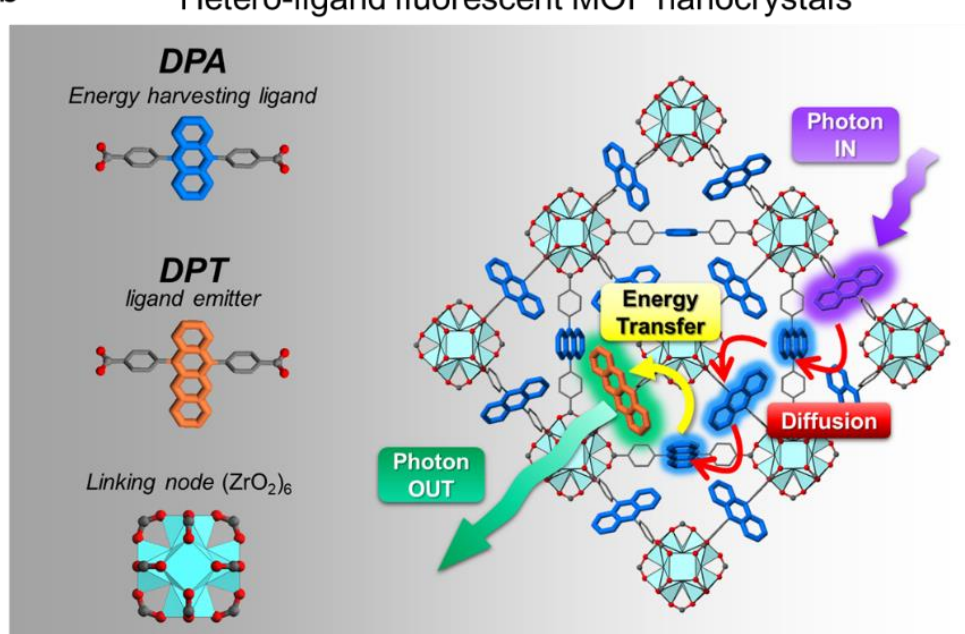

Figure 1 | Large Stokes shift emitters concept, applications and realization with engineered hetero-ligand MOF nanocrystals based on fluorescent ligands. a, Definition of large Stokes shift emitter. The energy difference between absorption and luminescence maxima $\Delta E$ is larger than luminescence half-height bandwidth $2 \sigma$, thus avoiding reabsorption. b. (left) Molecular structure of the MOF nanocrystals building blocks, namely the 9,10-diphenylantracenedicarboxylate fluorescent ligand (DPA), the 5,12-diphenyl-tetracenedicarboxylate fluorescent co-ligand (DPT) and the linking node zirconium oxo-hydroxy cluster. (right) Sketch of the energy flux after generation of a DPA singlet exciton, in this case upon absorption of a UV photon of energy $h v_{i n}$. The singlet exciton diffuses within the crystalline framework until it reaches the co-ligand DPT. The latter is excited by non-radiative energy transfer and then recombines radiatively by emitting a green photon of energy $h v_{\text {out }}$ with a Stokes shift $\Delta E=h v_{\text {in }}-h v_{\text {out }}$.

The extensive recent literature in the field of semiconductor nanocrystals testifies this very actual interest on large Stokes shift emitters. In these materials, for example, the $\Delta E$ can be tuned by doping of semiconductor with electronic impurities, ${ }^{11}$ resulting in the appearance of intragap states from which red shifted luminescence is produced. A notable $\Delta E$ value as large as $1 \mathrm{eV}$ can be achieved, ${ }^{12}$ 
but a current unsolved drawback is the slow luminescence kinetics that strongly limit their use for fast timing applications in nanosecond time scale and below. ${ }^{10,13,14}$ Moreover, in photonic devices where fast timing is foreseen, traditional wavelength shifters exploiting radiative energy transfer cannot be employed, because of the consequent slowing down of emitted light pulse.

In the search for fast emitters with remarkably large Stokes shift, we selected Metal-Organic Frameworks (MOFs), which constitute a solid platform to build materials wherein active struts perform tailored functions. Synthetic procedures based on self-assembly processes enable the controlled framing of struts in the porous crystalline architecture and the regulation of distances among linkers. ${ }^{15,16}$ The impressive versatility of MOFs promoted several applications such as gas storage, ${ }^{17,18}$ catalysis and dynamic materials, ${ }^{19-23}$ and triggered the most recent advances in the field of luminescent MOFs. ${ }^{24-29}$ This hot topic gave birth to a new class of optically active nanomaterials with tailorable electronic properties for photonics and optoelectronics, sensing, and biomedicine. 26,30,31 MOFs are also excellent candidates to be used in light-emitting devices, due to their structural diversity and tunable emission. A key advantage is the possibility to design their framework composition and structure which control both optical and energy-transport properties, such as those required for managing site specific photoreactions, ${ }^{32}$ or multi-excitonic processes. ${ }^{33}$ Therefore, optimized luminescent MOF nanocrystals can represent the next generation of luminescent materials with a potential impact comparable to their inorganic counterpart colloidal semiconductor nanocrystals.

Among conjugated molecules, polycyclic aromatic hydrocarbons of acene family have attracted great interest for various application in photon managing such as photon upconversion and singlet fission because of their peculiar electronic properties. ${ }^{34-37}$ Here we present the fabrication of MOF nanocrystals with fine-tuned composition, wherein tetracene-bearing fluorescent moieties were co-assembled with anthracene-based linkers to engineer the system emission properties and obtain significant energy down conversion of the emitted photons with respect the absorption, thus maximizing the emission Stokes shift. MOFs containing linear tetracene linkers have not yet been realized so far. The strategy of increasing the number of fused aromatic rings in the ligand core, yet maintaining a constant spacing between the chelating groups, proved successful in providing a series of customized hetero-ligand Zr-MOFs, which exhibited benchmark-efficiency fluorescence accompanied with negligible reabsorption. MOF nanocrystals were obtained by co-assembling the green-fluorescent chromophore 5,12-diphenyl-tetracenedicarboxylate (DPT) and the blue-emitting ligand 9,10-diphenyl-anthracenedicarboxylate (DPA) with $\mathrm{Zr}$ oxy-hydroxy nodes (Fig.1b). By exploiting the diffusion within the crystalline framework of singlet molecular excitons generated on DPA ligands, the incorporated DPT co-ligands are excited by means of non-radiative energy transfer and subsequently recombine radiatively producing photons with a $\Delta E$ as large as $750 \mathrm{meV}$. The fine matching of frequency emission of antracene moieties with the absorption of tetracene units enables an efficient energy transfer (ET) of $97 \%$ and photoluminescence quantum yield (QY) of $~ 70 \%$ even with a low DPT loading of $8 \%$ with respect to DPA (denoted Zr-DPT:DPA-8\%). Such a low loading enables to preserve the structural features of the parent homo-ligand nanocrystals. The potential technological transfer of the obtained hetero-ligand fluorescent nanomaterials is demonstrated by the realization of a prototypal fast polymeric nanocomposite scintillator that shows enhanced performances with respect to the homo-ligand nanocrystals, thus achieving benchmark values competing with those of several organic and inorganic commercial systems.

We designed and prepared the new conjugated tetracene-containing ligand (DPT) to be coassembled with the antracene-based linker (DPA) by a solvothermal process (Methods and Supplementary Information): the two molecules DPA (QY=0.96) and DPT (QY=0.80) were chosen 
because of their complementary of absorption/emission properties that make them an ideal donor (DPA) and acceptor (DPT) pair for non-radiative energy transfer (Supplementary Information). The two rod-like ligands with identical end-to-end length and connectivity were co-assembled by zirconium oxy-hydroxy clusters, generating a series of isostructural hetero-ligand MOF nanocrystals with modulated composition ranging from $0.1 \%$ to $8 \%$ of DPT/DPA ligand ratio content $(\mathrm{Zr}-$ DPT:DPA-x\%, Fig.1b). For comparison, the homo-ligand MOFs were synthetized using separately the single ligands (Zr-DPA and Zr-DPT, respectively). The composition of hetero-ligand Zr-MOFs is in agreement with the feeding ratio, as shown by ${ }^{1} \mathrm{H}$ NMR of digested samples. Connectivity, purity, and thermal stability were demonstrated by FT-IR, ${ }^{13} \mathrm{C}$ MAS NMR and TGA analysis (Supplementary Figs. S1-S40). Scanning electron microscopy (SEM) images of the Zr-DPT:DPA specimens reveal a homogenous population of nanocrystals with octahedral morphology. Figure 2a depicts the SEM image of the Zr-DPT:DPA-8\% sample, which consists in a nanocrystal ensemble with average size of $185 \pm 20 \mathrm{~nm}$.
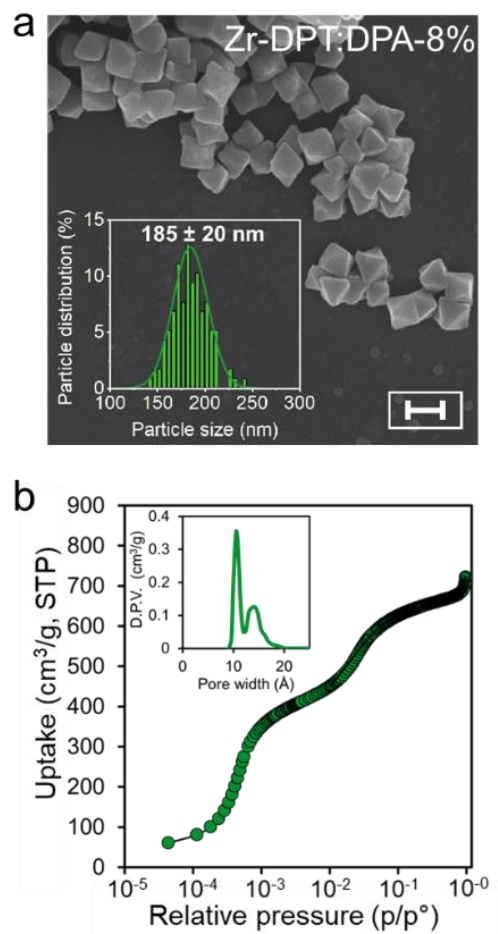

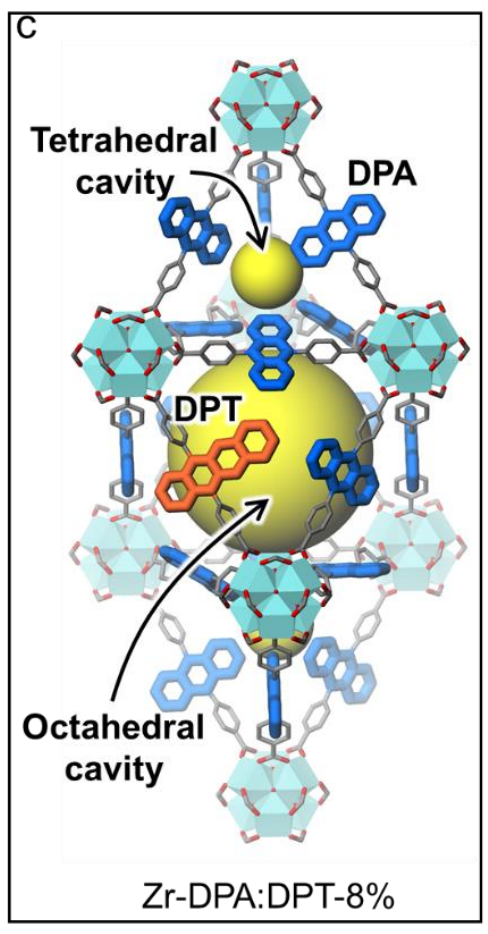

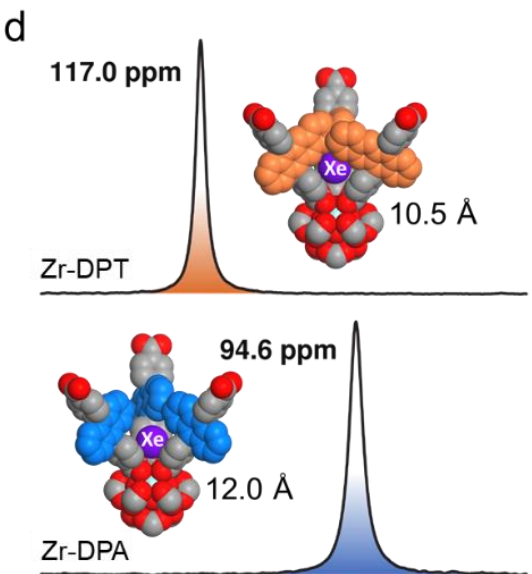

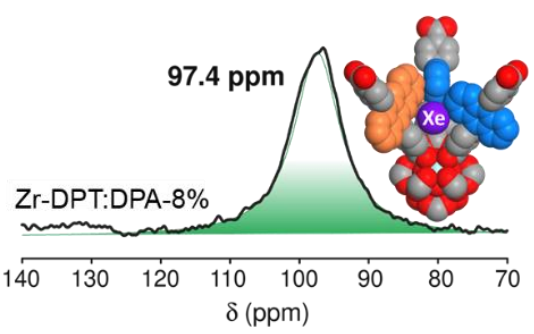

Figure 2 | Hetero-ligand zirconium-based metal-organic framework (MOF) nanocrystals structural properties. a, Scanning electron microscopy image of MOF nanocrystals realized with a DPT/DPA ligand ratio of 8\% (Zr-DPT:DPA$8 \%$ ). The inset reports the distribution of MOF size (Scale bar: $200 \mathrm{~nm}$ ). b, $\mathrm{N}_{2}$ adsorption isotherm collected at $77 \mathrm{~K}$. The inset showed the pore size distribution with two distinct cavities of $10.8 \AA$ and $14.5 \AA$. c, Crystal structure of $\mathrm{Zr}$-DPT:DPA highlighting the tetrahedral and octahedral cavities (yellow spheres). d, Hyperpolarized laser-assisted ${ }^{129}$ Xe NMR spectra of hetero-ligand nanocrystals compared with homo-ligand MOFs based on DPA and DPT.

Upon activation at $130^{\circ} \mathrm{C}$ under vacuum, the hetero-ligand $\mathrm{Zr}-\mathrm{MOF}$ nanocrystals exhibit a high crystallinity and a cubic crystal structure $(F m-3 m)$ with fcu topology, as established by PXRD Rietveld refinement, which corresponds to that of parent Zr-DPA and Zr-DPT MOFs (Supplementary Figs. S13-S17). Twelve ligands coordinate to each $\mathrm{Zr}$-based node $\left(\mathrm{Zr}_{6}\left(\mu_{3}-\mathrm{O}\right)_{4}\left(\mu_{3}-\mathrm{OH}\right)_{4}\left(\mathrm{CO}_{2}\right)_{12}\right.$ cluster) and yield a framework containing interconnected octahedral and tetrahedral cavities (Fig.2c). Thus, the ligands are arranged at a sufficiently short center-to-center distance of $11.7 \AA$ that enables both fast exciton diffusion and non-radiative energy transfer (Supplementary Information). Consistently with the crystal structure, $\mathrm{N}_{2}$ adsorption isotherms at $77 \mathrm{~K}$ (Fig. 2b) showed remarkable surface areas up to $3000 \mathrm{~m}^{2} / \mathrm{g}$ and step-wise profiles due to the subsequent filling of the well- 
differentiated tetrahedral and octahedral cavities with pore size of $10.8 \AA$ and $14.5 \AA$ in the framework. The size, symmetry and homogeneity of the pores were probed by the highly sensitive hyperpolarized laser-assisted ${ }^{129} \mathrm{Xe}$ NMR (Fig. 2d). The homo-ligand Zr-DPA and Zr-DPT MOFs show a single sharp signal at $\delta=94.6$ and $\delta=117.0 \mathrm{ppm}$, respectively, reflecting the patency of the cavities and the smaller size in Zr-DPT caused by the long flag-like conjugated tetracene moiety protruding into the nanochannels, with a steric encumbrance larger than that of antracene unit. The hetero-ligand Zr-DPT:DPA-8\% MOF exhibits a chemical shift at $\delta=96.5 \mathrm{ppm}$ which corresponds precisely to the expected weighted-average of the two chemical shifts of the homo-ligand nanocrystals. Remarkably, no residual signals of the homo-ligand MOFs are present, demonstrating the excellent structural homogeneity of the co-assembled nanocrystal ensemble.

The photophysical properties of the obtained MOF nanocrystals are investigated by means of photoluminescence spectroscopy. Figure 3a shows the optical absorption and continuous wave photoluminescence spectra of the hetero-ligand Zr-DPT:DPA-1\% in tetrahydrofuran dispersion $(0.1$, $\left.\mathrm{mg} \mathrm{mL}^{-1}\right)$. We observe a main absorption band in the near-UV spectral matching the profile of the Zr-DPA reference sample, because the absorption of the few DPT substituents is negligible. Upon photoexcitation at $355 \mathrm{~nm}$, the hetero-ligand nanocrystals show a broad luminescence in the visible spectrum. The most intense emission peaked at $515 \mathrm{~nm}$ and its vibronic replicas at $550 \mathrm{~nm}$ and 590 $\mathrm{nm}$ match the photoluminescence profile of the isolated DPT ligand (Fig.S7) and of the Zr-DPT MOF as a control sample. MOFs. This result suggests that the green luminescence is generated by the radiative recombination of singlet excitons on DPT co-ligands populated by energy transfer from directly excited DPAs ligands, as demonstrated by the excitation photoluminescence spectrum recorded at $540 \mathrm{~nm}$ that follows the DPA absorption profile (Fig.3a). The weak residual blue luminescence peaked at $430 \mathrm{~nm}$ mirrors an energy transfer yield $\left(\phi_{E T}\right)$ lower than unity. Nevertheless, the presence of this residual emission is crucial to investigate the antenna effect sketched in Fig.1b that occurs in the framework. Figure $3 \mathrm{~b}$ depicts the normalized photoluminescence spectra of the $\mathrm{Zr}$ DPT:DPA-1\% dispersion at different dilution ratios. Notably, the relative intensity of the green and blue emission components is unchanged. This is a crucial result, because if DPAs and DPTs experienced radiative energy transfer as two separated entities, i.e. in a standard bicomponent solution, the $\phi_{E T}$ value should decrease following the solution dilution level that reduces the concentration of the energy acceptor DPT and therefore the transfer probability. ${ }^{1,38}$ Consequently, the relative intensity of the green vs. blue component should be reduced as well. Conversely, the data clearly indicate that $\phi_{E T}$ is independent from the DPT dilution, thus demonstrating that each nanocrystal works as an individual emitter whose green luminescence is activated by intra-crystal energy transfer.

This picture is confirmed by time resolved experiments. The top inset of Fig. $3 \mathrm{~b}$ shows the photoluminescence intensity decay with time at $430 \mathrm{~nm}$ as a function of the dilution level. The signal decay in the hetero-ligand MOF is faster than in the Zr-DPA reference, indicating an efficient energy transfer (vide infra), ${ }^{1}$ but still no change in the decay kinetic is observed at different dilutions, thus demonstrating that the DPA-DPT interaction is unaffected. Similarly, the bottom inset shows that the green photoluminescence intensity at $540 \mathrm{~nm}$ decays with time as a single exponential function with a characteristic lifetime of $\tau \sim 11 \mathrm{~ns}$ (Supporting Table 7) regardless of the dilution level. This is another key result, indeed, the observed lifetime is almost identical to that one of the DPT molecule (11.5 ns, Fig.S41), and is significantly longer with respect to the reference homo-ligand DPT-MOFs (7.7 ns, Supporting Table 7). These findings suggest that co-assembled DPT ligands are effectively incorporated and framed as non-interacting single molecules within the nanocrystal architecture and preserve their excellent luminescence properties pivotal for the fabrication of photonic devices. 

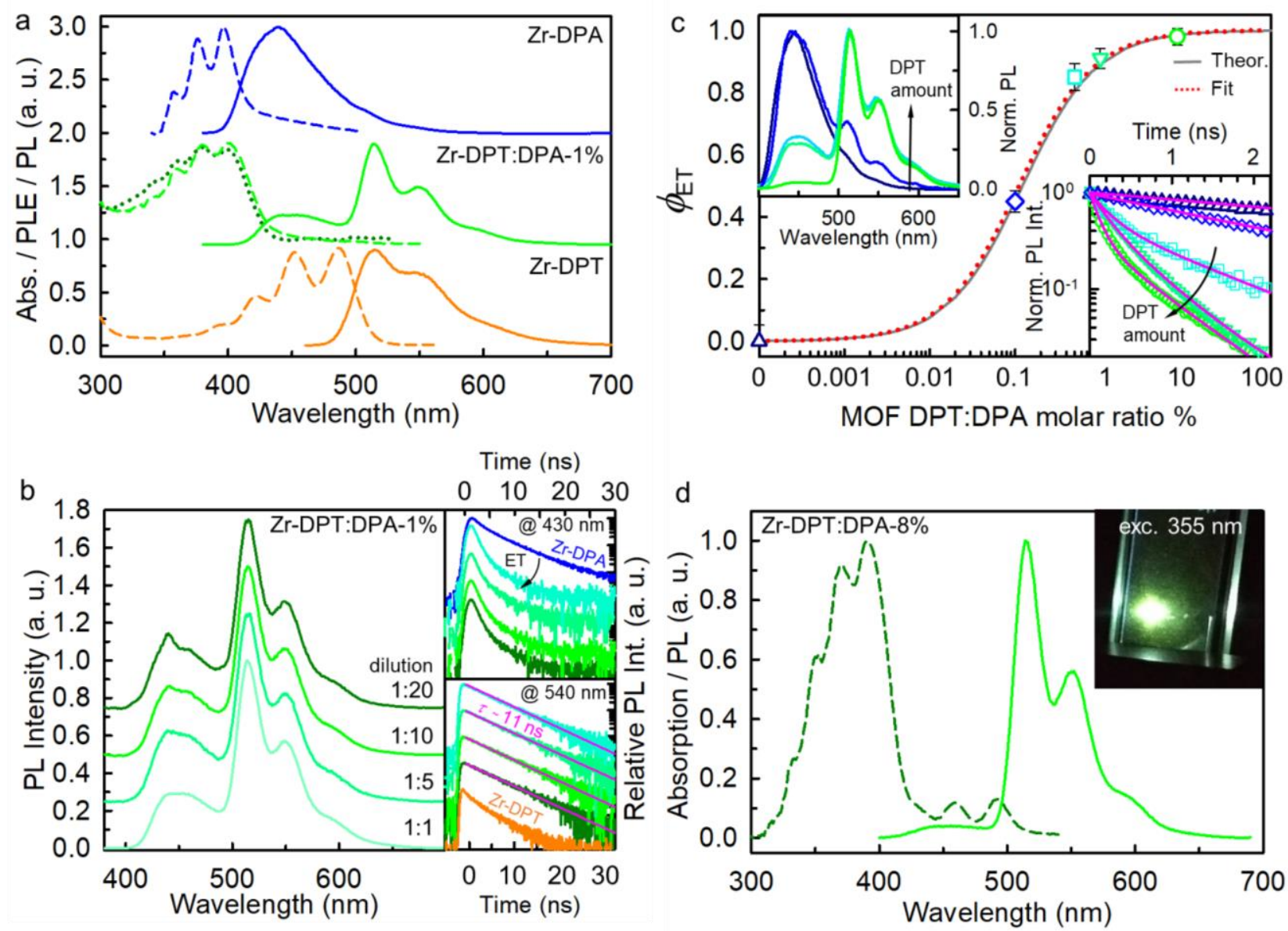

Figure 3 | Photophysical properties of hetero-ligand MOF nanocrystals. a, Normalized absorption (dashed line) and photoluminescence (PL, solid line) spectra of the hetero-ligand Zr-DPT:DPA-1\% nanocrystals $\left(0.1 \mathrm{mg} \mathrm{mL}^{-1} \mathrm{in}\right.$ tetrahydrofuran) compared with homo-ligand MOFs based on DPA (Zr-DPA, $0.1 \mathrm{mg} \mathrm{mL}^{-1}$ ) and DPT (Zr-DPT, $0.1 \mathrm{mg}$ $\left.\mathrm{mL}^{-1}\right)$. The excitation wavelength is $405 \mathrm{~nm}$. The dotted line is the excitation photoluminescence spectrum of the heteroligand MOF dispersion recorded at $550 \mathrm{~nm}$ b, PL spectrum of the Zr-DPT:DPA-1\% dispersion at different dilution ratios under 355 laser excitation. The insets show the PL intensity decay with time at $430 \mathrm{~nm}$ (top) and $540 \mathrm{~nm}$ (bottom) in comparison with that one of Zr-DPA and Zr-DPT reference nanocrystals, respectively. c, Energy transfer efficiency $\phi_{E T}$ between DPA and DPT co-ligands as a function of their relative molar ratio in the MOF framework. The theoretical $\phi_{E T}$ (solid line) is calculated considering a diffusion-mediated Förster energy transfer with interaction radius of $2.8 \mathrm{~nm}$ derived from ligands properties. The fit of experimental data (dotted line) results a characteristic interaction radius of $2.9 \mathrm{~nm}$. The top inset shows the PL spectra of the series of nanocrystals dispersions analyzed $\left(0.1 \mathrm{mg} \mathrm{mL}^{-1}\right)$. The bottom inset show their time-resolved PL spectra recorded at $430 \mathrm{~nm}$. Solid lines are the fit of the data with multi-exponential decay function. d, Absorption (dashed line) and PL (solid line) spectrum of Zr-DPT:DPA-1\% nanocrystals dispersion $\left(0.1 \mathrm{mg} \mathrm{mL}^{-1}\right.$, optical path $1 \mathrm{~cm}$ ). The excitation wavelength is $355 \mathrm{~nm}$. The inset is a digital picture of the dispersion in the quartz cuvette under $355 \mathrm{~nm}$ excitation.

Once assessed the validity of the synthetic strategy, we investigate quantitatively the energy transfer mechanism. As sketched in Fig.1b, the activation of DPT luminescence occurs by energy transfer during the random diffusion within the framework of an excited DPA singlet exciton, which is created upon light absorption or free-charge recombination in scintillation. ${ }^{39}$ Before spontaneous recombination, the singlet moves from the original position by an average diffusion length $L .{ }^{40-42}$ This implies that if a DPT moiety is placed at a distance shorter than $L$ from the position where the DPA exciton is created, the energy transfer can most likely occur before singlet recombination, thus without energy dispersion. Considering the MOF structure and a DPA-DPT Förster interaction radius of $R_{f s}=2.8 \mathrm{~nm}$ (Fig. S42), the theoretical energy transfer rate $k_{E T}$ and efficiency $\phi_{E T}$ are calculated as a function of the nanocrystal composition under the assumption of a diffusion-mediated energy 
process (Supplementary Material) ${ }^{38}$ The solid line in Fig. $3 \mathrm{c}$ depicts the theoretical $\phi_{E T}$ vs. the DPT ligand fraction in the hetero-ligand MOFs, expressed as the nominal DPT:DPA relative molar ratio employed for the synthesis. The plot shows that an excellent $\phi_{E T} \sim 0.9(90 \%)$ is reached with a DPT content as low as $1 \%$. This suggests that the proposed strategy to achieve large Stokes shift is already effective with low levels of DPT substitution in the DPA based MOF. In this way the risk to affect the MOF structural properties is minimized. The predicted $\phi_{E T}$ is compared with the one measured in the series of nanocrystals. The top inset of Figure $3 \mathrm{c}$ shows the normalized photoluminescence spectra of the MOF samples dispersed in tetrahydrofuran under $355 \mathrm{~nm}$ excitation. The blue emission from DPA ligands is almost completely switched off in the Zr-DPT:DPA-8\% sample. The experimental $\phi_{E T}$ value is calculated from the time resolved photoluminescence data shown in the bottom inset (Supplementary Information). The intensity of the blue luminescence at $430 \mathrm{~nm}$ decays as multi-exponential function (Supplementary Table 7), whose average lifetime shortens progressively by increasing the DPT amount, according to the corresponding increment of $k_{E T}$. The circles in the main panel mark the observed $\phi_{E T}$, which increases up to $97 \%$ in the Zr-DPT:DPA-8\% sample. The fit of data with a diffusion-mediated energy transfer kinetics results in an experimental interaction radius $R_{f s}^{*}=2.9 \mathrm{~nm}$ for the DPA/DPT pair, in perfect agreement with the proposed model. It is worth noting that the decay of the negligible residual blue emission intensity shows a biexponential behavior (Fig. 3c inset, Supplementary Information) that indicates the presence of two population of emitters. The fast component (67\% of the total signal) has a lifetime $\tau_{\text {fast }}=120 \mathrm{ps}$ $\approx\left[k_{E T}\right]^{-1}$ and accounts for the fraction of fully active large Stokes shift nanocrystals $\left(\phi_{E T}=1\right)$. The slow component is instead ascribed to a small sub-population of nanocrystals in the ensemble, statistically-containing a lower amount of DPT, where the transfer is not complete $\left(\phi_{E T}<1\right)$. From this analysis it is straightforward to estimate the photoluminescence quantum efficiency of fully active nanocrystals as $\mathrm{QY}=\mathrm{QY}{ }^{\text {ens }} / 0.67=0.69 \pm 0.06$, where $\mathrm{QY}{ }^{\text {ens }}=0.46 \pm 0.05$ is the photoluminescence yield measured for the ensemble in dispersion (Fig. 3d, Methods). Therefore, a huge photoluminescence Stokes shift of $750 \mathrm{meV}$ with highly efficient emission is achieved while preserving the fast time response that outperforms the one obtained in doped semiconductor quantum dots. ${ }^{43}$ Notably, both fast energy transfer and high yield Stokes shifted fluorescence are achieved by using low DPT amounts, thanks to the accurate design of hetero-crystals made by co-ligands with identical length and connectivity but strictly complementary optical properties owing to the change of their conjugation length. In such a way, the fast singlet excitons diffusion of the homo-ligand MOFs is unaffected by the presence of the acceptor moiety in the hetero-ligand structure, enabling ultrafast activation of the low energy emitters. The results obtained highlight therefore once more the potential of composition-tuned MOFs to develop efficient loss-free energy harvesting and transport systems that can mimic, for example, also natural photosynthetic mechanisms where fast energy migration is required.

Given their excellent luminescence properties, we tested the hetero-ligand MOF nanocrystals as emitters in bulk scintillating devices typically employed as detector of ionizing radiation where large Stokes shift is usually required to maximize the extraction of scintillation light. ${ }^{8}$ Figure 4 a shows the radioluminescence spectrum under soft X-ray exposure of a composite scintillator (thickness 0.1 $\mathrm{cm}$, diameter $1 \mathrm{~cm}$ ) fabricated by loading Zr-DPT:DPA-8\% nanocrystals in a polydimethylsiloxane (PDMS) matrix ( $0.5 \%$ weight, Methods, Figs.S43-S46). The radioluminescence spectrum (solid line) is dominated by a structured green emission peaked at $530 \mathrm{~nm}$ with a weak residual emission at 430 $\mathrm{nm}$, suggesting that the scintillation light is produced by radiative recombination of DPT singlet excitons. The absence of reabsorption is demonstrated by the possibility to clearly observe the first vibronic replica in the emission spectrum at $515 \mathrm{~nm}$, as in the diluted dispersion case, despite the high concentration of embedded nanocrystals (Fig. 3d). This result is in excellent agreement with the 
simulated emission spectrum (circles) calculated considering the light propagation in the device (Methods). Radioluminescence measurements under continuous irradiation up to around one hundred Gy demonstrate the emission stability and the absence of long-time phosphorescence due to delayed carrier recombination (inset of Fig.4a). No significant variation of the radioluminescence intensity can be observed by heating the sample up to $50{ }^{\circ} \mathrm{C}$, demonstrating a good thermal stability (Fig.S47). After 30 days of exposure to the atmospheric moisture, a mere $10 \%$ reduction of the emission intensity is observed, demonstrating a good resistance to molecular oxygen despite tendency of tetracene to photobleaching (Fig.S48).
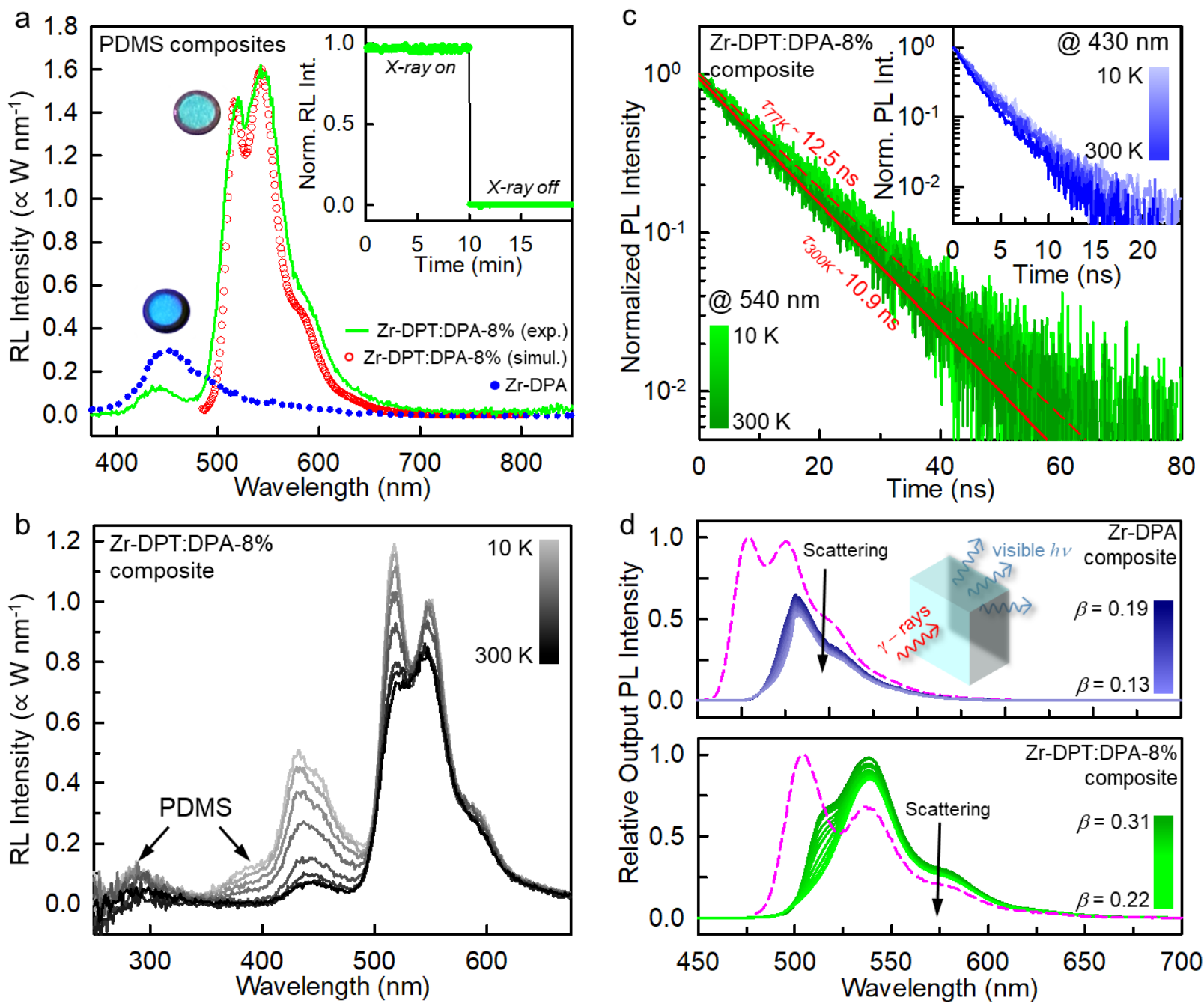

Figure 4 | Luminescence properties of large Stokes shift MOF-based nanocomposites under X-ray exposure. a, Experimental and simulated radioluminescence (RL) spectrum of nanocomposites based on loaded with hetero-ligand Zr-DPT:DPA-8\% and homo-ligand Zr-DPA nanocrystals fabricated by using polydimethylsiloxane (PDMS) as host polymer matrix $(0.5 \% \mathrm{wt}, 1 \mathrm{~mm}$ thickness) under X-ray exposure (digital pictures). RL intensity as a function of time (inset). b, RL spectrum of the Zr-DPT-DPA-8\% nanocomposite as a function of the temperature. c, Zr-DPT-DPA-8\% nanocomposite photoluminescence (PL) intensity decay recorded at $530 \mathrm{~nm}$ and $430 \mathrm{~nm}$ (inset) as function of the temperature under pulsed laser excitation at $405 \mathrm{~nm}$. d, Simulated output emission spectrum and efficiency $(\beta)$ as a function of the scattering level (from $0 \mathrm{~cm}^{-1}$ to $20 \mathrm{~cm}^{-1}$ ) in a $10 \times 10 \times 5 \mathrm{~mm}$ scintillator. Dashed lines depict the single molecule spectrum of the DPA (top) and DPT (bottom) chromophores for reference.

The nanocomposite shows a radioluminescence intensity five times greater than the reference composite made with the homo-ligand Zr-DPA (dotted line) which demonstrated a scintillation yield $\phi_{\text {scint }}$, defined as the number of emitted photons for each $\mathrm{MeV}$ of deposited energy for ionizing radiation, of approximately $1000 \mathrm{ph} \mathrm{MeV}^{-1} .{ }^{39}$ The obtained data indicates therefore that the prototype 
$\phi_{\text {scint }}$ is assessed at around $5000 \mathrm{ph} \mathrm{MeV}^{-1}$. This value demonstrates the success of the proposed strategy to enhance the scintillation performance of composite materials based on fluorescent MOF nanocrystals, which show a $\phi_{\text {scint }}$ comparable to that of commercial plastic an inorganic scintillators. ${ }^{45}$

We further investigated the composite emission by time resolved photoluminescence and radioluminescence experiments as function of the temperature. At room temperature the composite photoluminescence intensity at $540 \mathrm{~nm}$ decays with a characteristic lifetime of $10.9 \mathrm{~ns}$ (Fig.4c) matching that of the single molecules (Fig.4b). This finding is crucial to point out an important feature of the hetero-ligand emitters. In the case of homo-ligand MOFs, the use of high loading levels induces a shortening of the photoluminescence lifetime and efficiency reduction, because of a partial aggregation of poorly dispersed crystals that can limit also the surface passivation effect of the host matrix. ${ }^{39}$ Conversely, in hetero-ligand MOFs this effect appears absent, suggesting that DPT ligands are successfully incorporated as separated and protected units, whose emission ability is insensitive to the nanocrystal environment and aggregation-induced losses. ${ }^{39,46}$ The absence of significant reabsorption is further highlighted by low temperature experiments. By cooling the composite down to $10 \mathrm{~K}$, we still observe the first vibronic replica together with a simultaneous increment of the global green emission intensity ( $+19 \%$, Fig.4b). This increment is completely ascribed to the suppression of the intramolecular vibrational quenching mechanism at low temperature, as indicated by the emission lifetime that increases up to $12.5 \mathrm{~ns}$ at $10 \mathrm{~K}(+15 \%$, Fig. $4 \mathrm{c})$, thus demonstrating the absence of reabsorption-related losses. A more peculiar dynamic is observed for the radioluminescence blue component peaked at $430 \mathrm{~nm}$. At $10 \mathrm{~K}$, we observe a refinement of the vibronic structure in the residual DPA emission, as well as the expected slight lifetime increment (Fig.4b, inset) ${ }^{47}$ However, we observe the simultaneous growth of an overlapped component at $410 \mathrm{~nm}$, which is the main responsible of the blue emission intensity increment, and a UV emission peaked at $280 \mathrm{~nm}$ (Fig.S50). Both these components are competitive channels ascribed to the host PDMS. We notice that these electronic transitions are completely dark at $300 \mathrm{~K}$, thus we speculate that they can represent one of the main dissipative pathways that limit $\phi_{\text {scint }}$ due to a non complete energy transfer from the host matrix to the embedded nanocrystals.

The scintillation of large Stoke shift nanocomposites is investigated in a bulky cylindrical specimen (diameter $1 \mathrm{~cm}$, height $0.5 \mathrm{~cm}$ ) loaded with Zr-DPT:DPA-8\% ( $0.5 \%$ weight) irradiated with a pulsed X-ray beam (Methods). The expected emission output is reported in Fig.4d, obtained by simulating the propagation of photons in the composites including the scattering and reabsorption /reemission of travelling photons. The model reproduces a scintillation measurement where a photodetector is coupled by an index-matching on the largest face of the scintillator. The scattering is simulated as an artificial constant absorption background that is superimposed on the material absorption spectrum (Methods). The parameter $\beta$ is the geometrical detection efficiency, i.e. the fraction of scintillation photons that reaches the photodetector with respect the total number. The obtained results show that the efficiency of the scintillation light outcoupling at low scattering levels ( $\beta=0.31$ ) is significantly improved with respect to the composite based on the homo-ligand MOF Zr-DPA $(\beta=0.19)$. Notably, by exploiting the large Stokes shift nanoscintillators even in very extreme condition with a scattering as large as $20 \mathrm{~cm}^{-1}$, i.e. in the case of fabrication issues that result in an almost completely non-transparent item, the $\beta$ value assesses to 0.22 , thus highlighting the improvement achieved with the proposed material engineering strategy.

Fig.5a reports the $2 \mathrm{D}$ photoluminescence map of the specimen. Both the spectrum and the photoluminescence intensity decay under pulsed excitation at $405 \mathrm{~nm}$ exhibit the same features of the nanocrystal dispersion, i.e. a main emission peak at 540 with lifetime $\tau_{p l}=10.9 \mathrm{~ns}$ and a residual 
faster and weaker blue emission showing a multi-exponential decay behavior that reaches the ns time scale. The 2D scintillation map is showed in Fig.5b. The emission spectrum is analogous to the photoluminescence, with an intense scintillation band peaked at $540 \mathrm{~nm}$ and a weak blue emission at $430 \mathrm{~m}$ and same recombination kinetics. More precisely, the green scintillation characteristic lifetime $\tau_{\text {scint }}=10.4 \mathrm{~ns}$ matches well the $\tau_{p l}$ value, thus demonstrating that the MOFs preserve their excellent emission properties also if used as nanoscintillators. Taking a closer look to the ultrafast time scale, we observe that the green scintillation flash is well reproduced by a pulse function with an average decay time $\tau_{\text {decay }}=10.45=\tau_{\text {scint }}$ ns convoluted with the instrumental response function (Methods, inset of Fig. 5b). The scintillation pulse shows a rise time $\tau_{\text {rise }}$ of $190 \mathrm{ps}$, defined as the time variation between $10 \%$ and $90 \%$ of the maximum pulse intensity. Considering the speed of the energy transfer observed, an accurate estimate of the real rise time is therefore partially hindered by the instrumental response. However, no slow rise time is observed, thus demonstrating that the green scintillation is activated by the fast non-radiative energy transfer, with no contribution from the residual slow blue emitters.

a
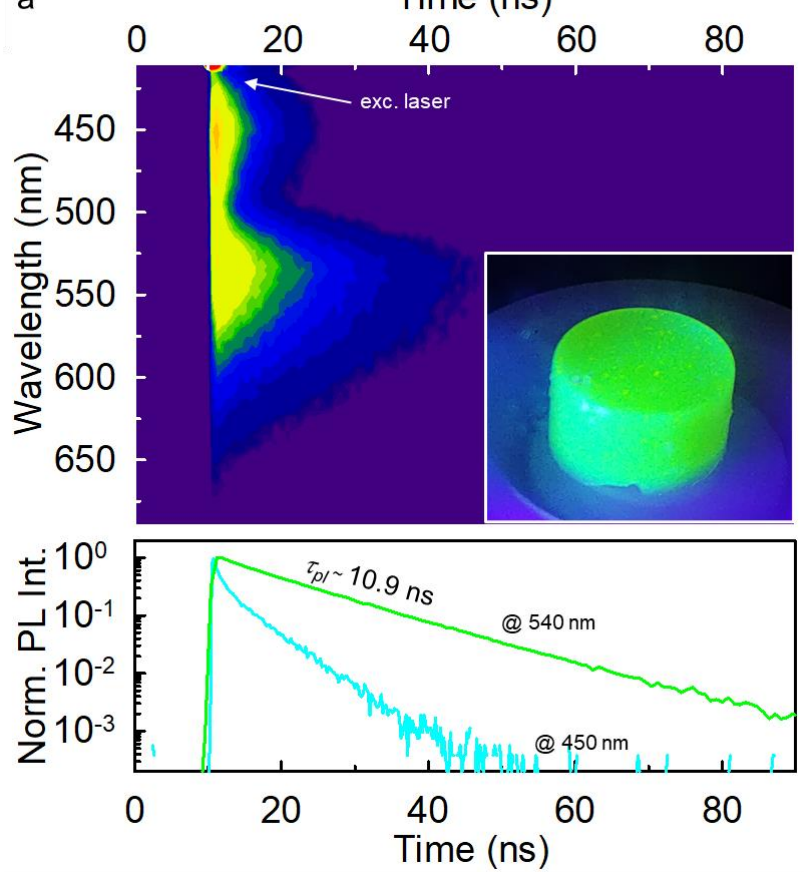

b
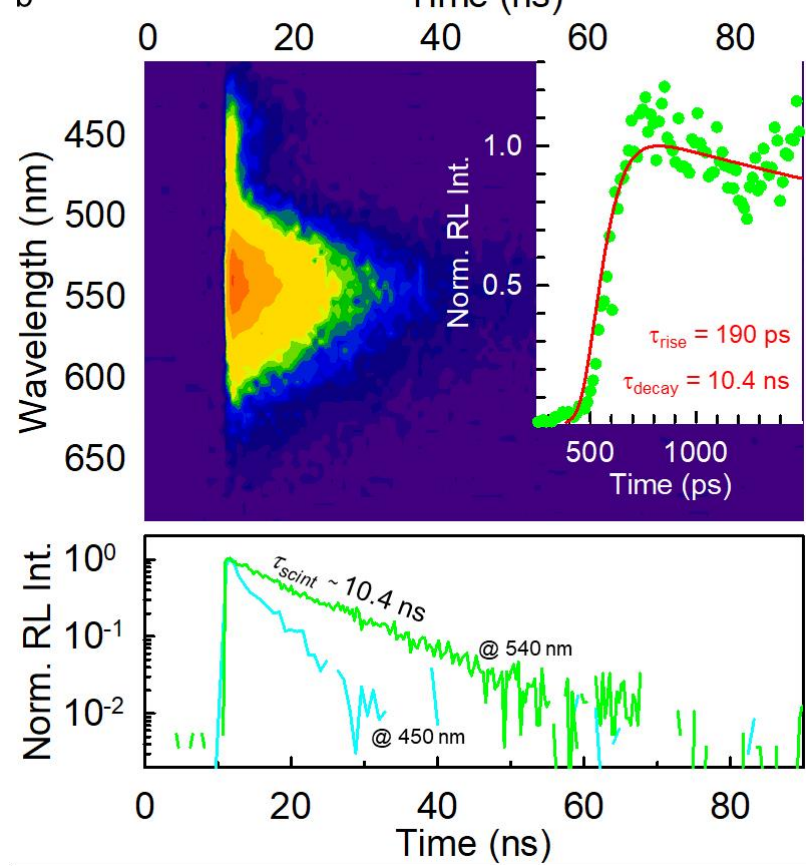

Figure 5 | Scintillation properties of large Stokes shift MOF-based nanocomposites. a, 2D map of the time resolved photoluminescence (PL) of the composite under 405 pulse laser excitation. The inset is a digital picture of the composite under UV lamp exposure. The bottom panel shows the decays in time of the PL intensity recorded at $540 \mathrm{~nm}$ and $430 \mathrm{~nm}$. b, 2D map of the composite scintillation emission under pulsed X-rays exposure. The bottom panel highlight the decay in time of the scintillation pulse intensity recorded at $540 \mathrm{~nm}$ and $430 \mathrm{~nm}$. The top panel inset shows the scintillation pulse recorded in the ultrafast time scale with the instrumental response function (IRF) and the fit of the data with a pulse function convoluted with the IRF with a given average decay time $\tau_{\text {decay }}$ of $10.4 \mathrm{~ns}$.

These findings demonstrate the success of the designed strategy of mixed acenes in MOFs and, despite the scintillation rise time cannot be accurately quantified, they suggest that even faster activation kinetics can be achieved. For example, by employing complementary ligands with better energetic resonance or by developing high-diffusivity nanocrystals by the fine tuning of the intermolecular orientation in the MOF framework, it would be possible to further enhance the energy transfer rate, thus achieving the activation of the Stokes shifted luminescence in times below the 100 ps threshold. These emitters will be therefore the ideal building blocks to realize high-optical quality bulk composite systems exploiting optimized loading strategies, which will enable to increase the 
amount of embedded nanoscintillators avoiding aggregation and therefore limiting the scattering of the scintillation light also in large area devices.

In conclusion, we successfully engineered the composition of co-assembled hetero-ligand MOF nanocrystals obtaining efficient and fast emitters with a large Stokes shift. Thanks to the fast diffusion-mediated non-radiative energy transfer mechanism occurring in the highly ordered crystalline framework between the different species of fluorescent ligands, we achieved a massive Stokes shift of $750 \mathrm{meV}$. This makes these new nanoemitters ideal candidates for reabsorption-free photonic applications that require fast timing response, thus surpassing the current limitation of large Stokes shift semiconductor nanostructures. The excellent emission quantum yield observed is a direct consequence of the success of the synthetic strategy employed to couple complimentary, fluorescent acene-based building blocks in a MOF architecture. Indeed, the results obtained indicate that the controlled incorporation of the energy acceptor ligands not only does not perturb the structural properties and stability of the MOFs, but also protect the antracene-emitters from surface-related luminescence quenching observed in homo-ligand MOF. Moreover, this strategy allows to protect the emitting ligands from the external environment also in a composite preferred form that exploits a transparent polymer host matrix, overcoming the energy losses related to phase segregation and aggregation. The potential impact of these new nanoemitters is highlighted by the investigation of their scintillation properties. The composite plastic scintillator based on hetero-ligand MOFs shows a better scintillation performance with respect to the previously investigated homo-ligand nanocrystals, which already demonstrated interesting features for the development of a new family of fast scintillators. Considering that the proposed strategy can be in principle applied to different ligand pairs with resonant electronic energies to tune both the emission Stokes shift and the spectral working ranges (according to specific application requests), the obtained results strongly support the future development of ligand-engineered MOF nanocrystals for photonic and photo-chemical applications that require energy harvesting, site specific collecting as well as photon frequency manipulation. 


\section{Methods}

Synthesis of Zr-DPT. MOF nanocrystals were synthetized under solvothermal conditions modulated by acetic acid. The conditions were optimized to generate a highly crystalline sample. Briefly, $\mathrm{ZrCl}_{4}$, 5,12-bis(4-carboxyphenyl) tetracene (DPT) and acetic acid were dispersed in DMF (see SI for further details). The resulting mixture was heated at $120^{\circ} \mathrm{C}$ for 22 hours and the orange powder was filtered and washed with fresh solvent before activation at $130^{\circ} \mathrm{C}$ under high vacuum before characterization. The synthesis of DPT ligand is reported in Supporting Information.

Synthesis of Zr-DPT:DPA-x\%. Nanocrystalline samples of Zr-DPT:DPA-x\% were synthetized under solvothermal conditions modulated with acetic acid. $\mathrm{ZrCl}_{4}$ and a proper amount of 5,12-bis(4carboxyphenyl)tetracene (DPT) and 9,10-bis(4-carboxyphenyl) anthracene (see SI for further details) were dispersed in a mixture of DMF and acetic acid. The mixture was heated at $120^{\circ} \mathrm{C}$ for 22 hours and the yellowish powder was filtered and washed with fresh solvent before activation at $130^{\circ} \mathrm{C}$ under high vacuum.

Synthesis of Zr-MOF:PDMS composites. PDMS nanocomposites were prepared by dispersing MOF nanocrystals in a prepolymer mixture that was poured in a proper mould and cured at $60^{\circ} \mathrm{C}$ to obtain self-standing nanocomposites. The nanocomposites were obtained by the reaction of vinyl-terminated polydimethylsiloxane with polydimethylsiloxane-co-methylhydrosiloxane by thermal curing. The cross-linking reaction starting from the polymer terminals preserved the flexibility of the polymer chains and produced very low glass transition. ${ }^{48}$

Structure analysis and microscopy. The structure and composition of Zr-MOF nanocrystals and nanocomposites were determined by means of powder X-ray diffraction (PXRD) structure refinement, nuclear magnetic resonance (NMR) spectroscopy, Fourier - transform infrared (FT-IR) spectroscopy, thermogravimetric analysis (TGA), adsorption properties, helium picnometry, scanning electron microscopy (SEM) and transmission electron microscopy (TEM). Details on the instrumental setup and the measurement protocols are reported in the supplementary material file. The crystal structures were refined by the Rietveld method combined with molecular mechanics and plane-wave DFT calculations (see Supplementary Material).

Photoluminescence studies. Absorption spectra has been recorded with a Cary Lambda 900 spectrophotometer at normal incidence using quartz Suprasil cuvettes with $0.1 \mathrm{~cm}$ of optical path and an integrating sphere to eliminate scattering effects. Steady state photoluminescence (PL) spectra were acquired with a Variant Eclipse fluorimeter (bandwidth $1 \mathrm{~nm}$ ) using quartz Suprasil cuvettes with $1 \mathrm{~cm}$ of optical path. Time-resolved photoluminescence experiments in the nanosecond time scale have been made by using as excitation source a pulsed laser LED at $340 \mathrm{~nm}$ (3.65 eV, EP-LED 340 Edinburgh Instruments, pulse width $80 \mathrm{ps}$ ). Quartz Suprasil cuvettes with $0.1 \mathrm{~cm}$ of optical path has been used to study nanocrystals dispersions. The nanocomposites were excited with a pulsed laser at $405 \mathrm{~nm}$ (3.06 eV, EPL-405 Edinburgh Instruments, pulse width $90 \mathrm{ps)} \mathrm{to} \mathrm{avoid} \mathrm{direct} \mathrm{excitation} \mathrm{of}$ the host polymer matrix. Experiments in the picoseconds time scale have been made by using a doubled Ti:Sapph pulsed laser at $380 \mathrm{~nm}$ (3.65 eV, Coherent Mira 900, pulse width $150 \mathrm{fs})$ coupled to a streak camera Hamamatsu Synchroscan M1955. The PL quantum yields of DPT and MOF 8\% dispersion have been measured by relative methods as described in the Supplementary Material file. Measurements on composites as a function of the temperature were performed on films $0.1 \mathrm{~cm}$ thickness and diameter $1 \mathrm{~cm}$, mounting the sample in closed circle He cryostat with direct optical access.

Radioluminescence studies. Steady state RL measurements were carried out at room temperature using a homemade apparatus featuring, as a detection system, a liquid nitrogen-cooled, back- 
illuminated, and UV-enhanced charge coupled device (CCD) Jobin-Yvon Symphony II, combined with a monochromator Jobin-Yvon Triax 180 equipped with a 100 lines/mm grating. All spectra are corrected for the spectral response of the detection system. RL excitation was obtained by unfiltered X-ray irradiation through a Be window, using a Philips $2274 \mathrm{X}$-ray tube with tungsten target operated at $20 \mathrm{kV}$. At this operating voltage, a continuous X-ray spectrum is produced by a Bremsstrahlung mechanism superimposed to the $\mathrm{L}$ and $\mathrm{M}$ transition lines of tungsten, due to the impact of electrons generated through thermionic effect and accelerated onto a tungsten target. The dose rate was 0.2 Gy/s, evaluated by comparison with a calibrated ${ }^{90} \mathrm{Sr}-{ }^{90} \mathrm{Y}$ beta radioactive source and using optically stimulated luminescence emission from quartz crystalline powder (100 - $200 \mu \mathrm{m}$ grains). In order to record the PL measurements, the same acquisition system of RL measurements has been coupled to a $405 \mathrm{~nm}$ pulsed diode laser (EPL-405 Edinburgh Instruments) through a quartz optical fibre bundle allowing the illumination of the sample in the X-ray chamber.

Scintillation studies. Pulsed X-rays with energies up to $25 \mathrm{keV}$ were generated with a repetition rate of $1 \mathrm{MHz}$ by a picosecond diode laser at $405 \mathrm{~nm}$ (Delta diode from Horiba) focused on a X-ray tube (model N5084 from from hamamatsu). In the case of optical excitation, the same laser (405nm) was used. The resulting photons were collected by Kymera spectrograph (ANDOR) and detected by an hybrid PMT 140-C from Becker \& Hickl GmbH. For decay-time measurements, the photons were histogramed using a PicoHarp300 time-correlated single-photon counting (32ps time/bin) and for the time resolved spectra a MCS6A multiple-channel time analyser was used (800ps time/bin) Subnanosecond scintillation emission kinetics of the samples were measured with a Time Correlated Single Photon Counting (TCSPC) setup. As excitation source a pulsed X-Ray beam (X-ray Tube XRT N5084, Hamamatsu), with a continuous energy spectrum between 0 and $40 \mathrm{keV}$ and a mean energy of $9.15 \mathrm{keV}$, produced with a Pulse Diode Laser (PDL 800-B, PicoQuant) were used. The scintillation light was collected in reflection by a Hybrid Photomultiplier Tube (HPM 100-07, Becker \& Hickel), operating in TCSPC mode, and processed by an Amplifier and Timing Discriminator (model 9237, ORTEC). This processed HPM output signal was used as stop signal for a Time to Digital Converter (TDC xTDC4, chronologic), while the start signal was given by the external trigger of the PDL. An optical band-pass filter (450 nm with a FWHM of $40 \mathrm{~nm}$ ) was used, chosen accordingly to the emission spectrum of the samples, to cut observed air excitation by X-Ray. The scintillation pulse was fitted with a convolution between the Impulse Response Function (IRF) of the whole system with a full width at half maximum (FWHM) of $180 \mathrm{ps}$ and the intrinsic scintillation rate. ${ }^{9}$ Sub-nanosecond scintillation emission kinetics of the samples were measured with a Time Correlated Single Photon Counting (TCSPC) setup. As excitation source a pulsed X-Ray beam (Xray Tube XRT N5084, Hamamatsu), with a continuous energy spectrum between 0 and $40 \mathrm{keV}$ and a mean energy of $9.15 \mathrm{keV}$, produced with a Pulse Diode Laser (PDL 800-B, PicoQuant) were used. The scintillation light was collected in reflection by a Hybrid Photomultiplier Tube (HPM 100-07, Becker \& Hickel), operating in TCSPC mode, and processed by an Amplifier and Timing Discriminator (model 9237, ORTEC). This processed HPM output signal was used as stop signal for a Time to Digital Converter (TDC xTDC4, chronologic), while the start signal was given by the external trigger of the PDL. An optical band-pass filter (450 nm with a FWHM of $40 \mathrm{~nm}$ ) was used, chosen accordingly to the emission spectrum of the samples, to cut observed air excitation by X-Ray. The scintillation pulse was fitted with a convolution between the Impulse Response Function (IRF) of the whole system with a full width at half maximum (FWHM) of 180 ps and the intrinsic scintillation rate. ${ }^{9}$

Light propagation modelling. Simulations of the scintillating nanocomposite performances were carried out using a Monte Carlo ray-tracing method previously presented. ${ }^{39}$ The photon propagation follows geometrical optics laws where the interference is neglected. Each photon can be absorbed and re-emitted by a chromophore, isotropically scattered, and reflected or transmitted at the 
interfaces, where the Fresnel coefficients have been used to compute the reflection probability. The simulated scintillator contains the same number of nanocrystals employed to fabricate the described sample. The absorption, scattering, transmission, or reflection events are chosen according to random Monte Carlo drawing. The simulations were performed using the experimental absorption/luminescence spectrum and emission efficiency of nanocrystals $\left(\Phi_{\mathrm{PL}}=67 \%\right.$ for $\mathrm{Zr}$ DPT:DPA-8\% and $\Phi_{\mathrm{PL}}=27 \%$ for $\left.\mathrm{Zr}-\mathrm{DPA}\right)$. The scattering is supposed to induce light attenuation corresponding to an absorption coefficient ranging from 0 to $20 \mathrm{~cm}^{-1}$.

\section{Acknowledgements}

Financial support from the Italian Ministry of University and Research (MIUR) through grant "Dipartimenti di Eccellenza- 2017 "Materials for Energy" is gratefully acknowledged. The authors acknowledge support from PRIN-20173L7W8K. The work performed at CERN was made in frame of Crystal Clear Collaboration

\section{Author contributions}

J.P. and A.C. designed and synthesized the MOF nanocrystals and fabricated the composites. J.P., S.B., P.E.S., C.X.B. and A.C. analysed the nanocrystals and composite structural properties. I.V., R.C., F.C and A.V. performed the radioluminescence studies. C.D., I.F, N.K., F.P and EA performed the scintillation experiments. F.M. and A.M. performed and supervised the photoluminescence studies. F.M. and A.M. developed the numerical modelling of the nanocomposite optical properties. A. C. and A.M. conceived and designed the project.

\section{Competing financial interests}

The authors declare no competing financial interests.

\section{Additional information}

Supplementary information is available in the online version of the paper. Correspondence and requests for materials should be addressed to A. M.. 


\section{References}

1 Lakowicz, J. R. Principles of fluorescence spectroscopy. (Springer Science+Business Media, 2010).

2 Hehlen, M. P. Reabsorption artifacts in measured excited-state lifetimes of solids. J. Opt. Soc. Am. B 14, 1312-1318 (1997).

3 Yamada, T., Yamada, Y., Nakaike, Y., Wakamiya, A. \& Kanemitsu, Y. Photon Emission and Reabsorption Processes in $\mathrm{CH}_{3} \mathrm{NH}_{3} \mathrm{PbBr}_{3}$ Single Crystals Revealed by Time-Resolved Two-PhotonExcitation Photoluminescence Microscopy. Physical Review Applied 7, 014001 (2017).

4 Staub, F., Anusca, I., Lupascu, D. C., Rau, U. \& Kirchartz, T. Effect of reabsorption and photon recycling on photoluminescence spectra and transients in lead-halide perovskite crystals. Journal of Physics: Materials 3, 025003 (2020).

5 Zhang, J. et al. Highly Stable Near-Infrared Fluorescent Organic Nanoparticles with a Large Stokes Shift for Noninvasive Long-Term Cellular Imaging. ACS Applied Materials \& Interfaces 7, 2626626274 (2015).

6 Ren, T.-B. et al. A General Method To Increase Stokes Shift by Introducing Alternating Vibronic Structures. Journal of the American Chemical Society 140, 7716-7722 (2018).

$7 \quad$ Mattiello, S. et al. Chemically Sustainable Large Stokes Shift Derivatives for High-Performance Large-Area Transparent Luminescent Solar Concentrators. Joule 4, 1988-2003 (2020).

8 Wang, X. et al. Organic phosphors with bright triplet excitons for efficient X-ray-excited luminescence. Nature Photonics 15, 187-192 (2021).

9 Gundacker, S., Auffray, E., Pauwels, K. \& Lecoq, P. Measurement of intrinsic rise times for various L (Y) SO and LuAG scintillators with a general study of prompt photons to achieve 10 ps in TOFPET. Physics in Medicine \& Biology 61, 2802 (2016).

10 Lecoq, P., Korzhik, M. \& Vasiliev, A. Can Transient Phenomena Help Improving Time Resolution in Scintillators? IEEE Transactions on Nuclear Science 61, 229-234 (2014).

11 Viswanatha, R., Brovelli, S., Pandey, A., Crooker, S. A. \& Klimov, V. I. Copper-Doped Inverted Core/Shell Nanocrystals with "Permanent" Optically Active Holes. Nano Letters 11, 4753-4758 (2011).

12 Khan, A. H. et al. Tunable and Efficient Red to Near-Infrared Photoluminescence by Synergistic Exploitation of Core and Surface Silver Doping of CdSe Nanoplatelets. Chemistry of Materials 31, 1450-1459 (2019).

13 Pinchetti, V. et al. Excitonic pathway to photoinduced magnetism in colloidal nanocrystals with nonmagnetic dopants. Nature Nanotechnology 13, 145-151, doi:10.1038/s41565-017-0024-8 (2018).

14 Mondal, P., Chakraborty, S., Grandhi, G. K. \& Viswanatha, R. Copper Doping in II-VI Semiconductor Nanocrystals: Single-Particle Fluorescence Study. The Journal of Physical Chemistry Letters 11, 5367-5372 (2020).

15 Yaghi, O. M. et al. Reticular synthesis and the design of new materials. Nature 423, $705-714$ (2003).

16 Zhou, H.-C. J. \& Kitagawa, S. Metal-Organic Frameworks (MOFs). Chemical Society Reviews 43, 5415-5418 (2014).

17 Chen, K.-J. et al. Synergistic sorbent separation for one-step ethylene purification from a fourcomponent mixture. Science 366, 241-246 (2019).

18 Siegelman, R. L., Kim, E. J. \& Long, J. R. Porous materials for carbon dioxide separations. Nature Materials 20, 1060-1072 (2021).

19 Rosi, N. L. et al. Hydrogen Storage in Microporous Metal-Organic Frameworks. Science 300, 1127 (2003).

20 Dolgopolova, E. A., Rice, A. M., Martin, C. R. \& Shustova, N. B. Photochemistry and photophysics of MOFs: steps towards MOF-based sensing enhancements. Chemical Society Reviews 47, 4710-4728 (2018).

21 Bavykina, A. et al. Metal-organic frameworks in heterogeneous catalysis: recent progress, new trends, and future perspectives. Chemical Reviews 120, 8468-8535 (2020).

22 Perego, J. et al. Fast motion of molecular rotors in metal-organic framework struts at very low temperatures. Nature Chemistry 12, 845-851 (2020).

23 Danowski, W. et al. Visible-light-driven rotation of molecular motors in a dual-function metal-organic framework enabled by energy transfer. Journal of the American Chemical Society 142, 9048-9056 (2020).

24 Mezenov, Y. A., Krasilin, A. A., Dzyuba, V. P., Nominé, A. \& Milichko, V. A. Metal-organic frameworks in modern physics: Highlights and perspectives. Advanced Science 6, 1900506 (2019). 
Yin, H.-Q. \& Yin, X.-B. Metal-Organic Frameworks with Multiple Luminescence Emissions: Designs and Applications. Accounts of Chemical Research 53, 485-495 (2020).

26 Lustig, W. P. et al. Metal-organic frameworks: functional luminescent and photonic materials for sensing applications. Chemical Society Reviews 46, 3242-3285 (2017).

27 Castiglioni, F. et al. Modulation of porosity in a solid material enabled by bulk photoisomerization of an overcrowded alkene. Nature Chemistry 12, 595-602 (2020).

28 Jia, J. et al. Access to Highly Efficient Energy Transfer in Metal-Organic Frameworks via Mixed Linkers Approach. Journal of the American Chemical Society 142, 8580-8584 (2020).

29 An, Z. et al. Influence of Isomerism on Radioluminescence of Purely Organic Phosphorescence Scintillators. Angewandte Chemie (2021).

30 Kreno, L. E. et al. Metal-organic framework materials as chemical sensors. Chemical reviews $\mathbf{1 1 2}$, 1105-1125 (2012).

31 Lu, K. et al. Low-dose X-ray radiotherapy-radiodynamic therapy via nanoscale metal-organic frameworks enhances checkpoint blockade immunotherapy. Nature biomedical engineering 2, 600610 (2018).

32 Laurier, K. G. M. et al. Iron(III)-Based Metal-Organic Frameworks As Visible Light Photocatalysts. Journal of the American Chemical Society 135, 14488-14491 (2013).

33 Park, J., Xu, M., Li, F. \& Zhou, H.-C. 3D Long-Range Triplet Migration in a Water-Stable MetalOrganic Framework for Upconversion-Based Ultralow-Power in Vivo Imaging. Journal of the American Chemical Society 140, 5493-5499 (2018).

34 Ravetz, B. D. et al. Photoredox catalysis using infrared light via triplet fusion upconversion. Nature 565, 343-346 (2019).

35 Einzinger, M. et al. Sensitization of silicon by singlet exciton fission in tetracene. Nature 571, 90-94 (2019).

36 Ronchi, A. et al. High Photon Upconversion Efficiency with Hybrid Triplet Sensitizers by Ultrafast Hole-Routing in Electronic-Doped Nanocrystals. Advanced Materials 32, 2002953, (2020).

37 Budden, P. J. et al. Singlet exciton fission in a modified acene with improved stability and high photoluminescence yield. Nature Communications 12, 1527 (2021).

38 Stryer, L., Thomas, D. D. \& Meares, C. F. Diffusion-Enhanced Fluorescence Energy Transfer. Annual Review of Biophysics and Bioengineering 11, 203-222 (1982).

39 Perego, J. et al. Composite fast scintillators based on high-Z fluorescent metal-organic framework nanocrystals. Nature Photonics 15, 393-400 (2021).

40 Pope, M. \& Swenberg, C. E. Electronic processes in organic crystals and polymers. Vol. 39 (Oxford University Press on Demand, 1999).

41 Scholes, G. D. Long-Range Resonance Energy Transfer in Molecular Systems. Annual Review of Physical Chemistry 54, 57-87 (2003).

42 Mikhnenko, O. V., Blom, P. W. \& Nguyen, T.-Q. Exciton diffusion in organic semiconductors. Energy \& Environmental Science 8, 1867-1888 (2015).

43 Sahu, A. et al. Electronic Impurity Doping in CdSe Nanocrystals. Nano Letters 12, 2587-2594 (2012).

44 Hughes, K. E., Hartstein, K. H. \& Gamelin, D. R. Photodoping and Transient Spectroscopies of Copper-Doped CdSe/CdS Nanocrystals. ACS Nano 12, 718-728 (2018).

45 Turtos, R. M., Gundacker, S., Auffray, E. \& Lecoq, P. Towards a metamaterial approach for fast timing in PET: experimental proof-of-concept. Physics in Medicine \& Biology 64, 185018 (2019).

46 Monguzzi, A. et al. Highly Fluorescent Metal-Organic-Framework Nanocomposites for Photonic Applications. Nano Letters 18, 528-534 (2018).

47 Ware, W. R. \& Baldwin, B. A. Effect of Temperature on Fluorescence Quantum Yields in Solution. The Journal of Chemical Physics 43, 1194-1197 (1965).

48 Bosq, N., Guigo, N., Persello, J. \& Sbirrazzuoli, N. Melt and glass crystallization of PDMS and PDMS silica nanocomposites. Physical Chemistry Chemical Physics 16, 7830-7840 (2014). 